Chapter 10

\title{
A New webGIS Platform Dedicated to Forest Extreme Events in the Alps: Aims and Functionalities
}

\author{
Stefano Oliveri, Marco Pregnolato and \\ Giacomo Gerosa \\ Additional information is available at the end of the chapter \\ http://dx.doi.org/10.5772/56269
}

\section{Introduction}

Many recent studies [1-6] show that, in the next future, climate change could represent a relevant threat for the state of health of Alpine forests. As a consequence, Alpine communities could be constrained to face an increase of both the frequency and the severity of damaging events affecting their forests (fires, pests attacks, windthrows, heavy snows). Although not all extreme damage occurrences are a direct consequence of extreme meteorological events, many of them have been linked to climatic phenomena. In some cases the relation is absolutely immediate: the storms Vivian (1990) and Lothar (1999), e.g., produced huge windthrows damages to the Alpine forests mainly in Germany, Switzerland and France with millions cubic meters of timber affected. The well-known heat wave registered during summer 2003 produced, following the statistics, peaks in the frequency of forest fires both in the same season and in the next winter. Well the same, the extremely hot summer of 2003 has been correlated to several pests outbreaks $[7,8]$ and several studies are conjecturing the possible influences of climate change on forest pathology. Hypothesis have been made about effects on the plant chemical defense mechanisms and on pests range expansions and diapauses, showing both a direct influence of temperatures and droughts periods on the biological cycle of the agents and an impact over the physiology of weakened host trees. Moving from these considerations, when MANFRED project was conceived partners recognized the need for a tool aimed at enabling Alpine communities to share their knowledge on past extreme events, in order to facilitate the diffusion and the sharing of practices which can be applied to prevent those extreme events, mitigate their effects and recover after extreme damaging occurrences. Whilst the greatest part of MANFRED activities have been intended to model and predict the future, the activities related to the development of the webGIS platform implied to look at the past, 
with the aim to derive the lessons learnt from a set of past extreme occurrences and use them as a tool to better manage the future.

\section{Materials and methods}

From an operative point of view, the work has been developed through the following steps:

- census of the events, with a special focus on extreme occurrences;

- identification of a set of relevant occurrences, which have been investigated in depth using a case histories approach;

- publication of results on a webGIS platform (available at the Internet address www.manfredproject.eu/webgis) [9], giving open access to the whole set of data gathered and allowing to perform, on those data, both geographic and thematic queries.

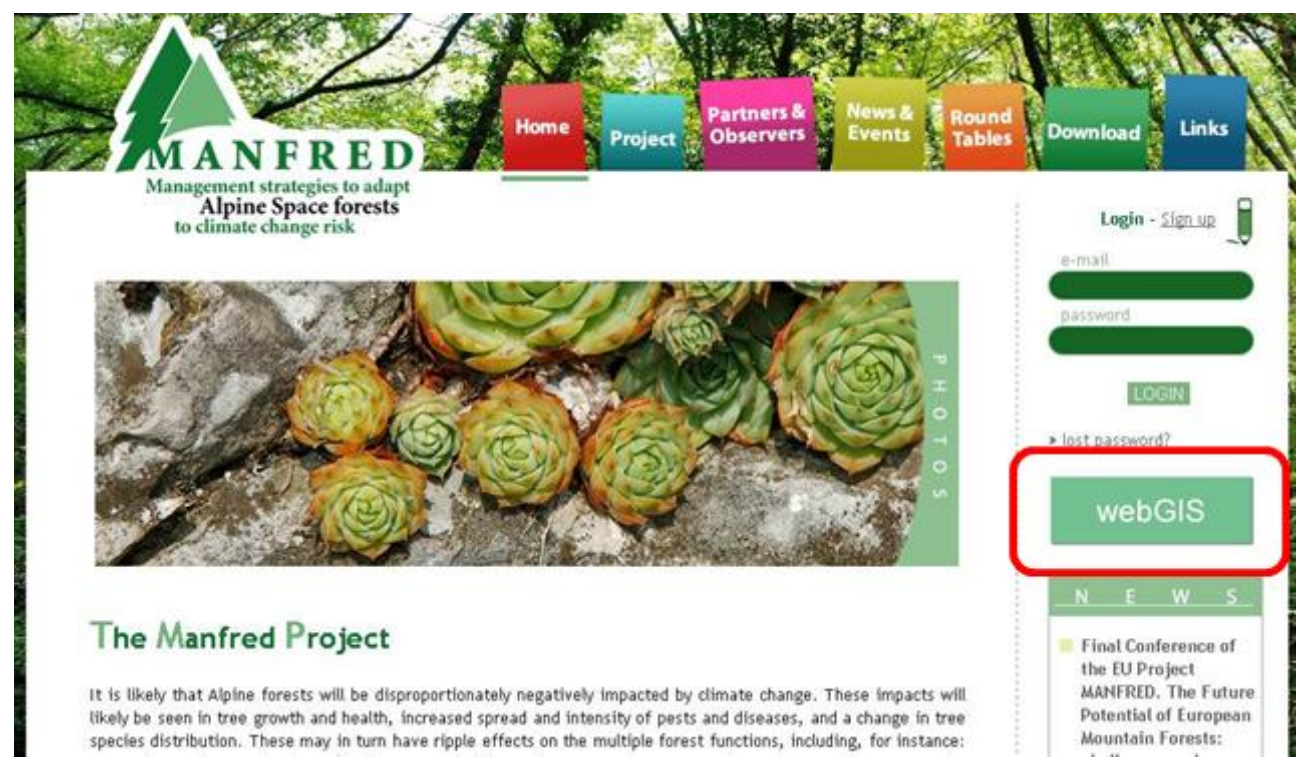

Figure 1. The project home page, with the button allowing the access to the webGIS platform

\subsection{Definition of extreme events}

The concept of extreme occurrence has to do with one's perception, familiarity and personal sensitivity. As a consequence, the definition of extreme event is associated with an intrinsic level of subjectivity. Yet, even sticking to the mere technical point of view, the interpretation of extreme stress event in a forest environment may dramatically depend on the different 
technical backgrounds and experience of the individuals giving the meaning. Foresters, landscape ecologist, conservationists, disaster and risk managers, statisticians and meteorologists would probably provide several keys, also referring to different forest-related benefits and roles. In fact, according to a multi-criteria and cross-sector approach, there could be more than one effective way to define an extreme event. Moreover, lack of numeracies and high heterogeneity of available data between the different Alpine regions, make it difficult the adoption of quantitative methods aimed at a standard identification of extreme occurrences. As a consequence, to warrant the selection of extraordinary events, a set of differentiated methods and approaches were applied based on the information and data available at the different areas of the Alpine arch. This kind of approach has been considered consistent with the aims of the activities carried out in the framework of MANFRED project, whose goal did not consist in a scientific definition of extreme event. It was considered much more suitable and useful to the aim of the project to describe an event as extreme mainly following the characteristic of being a non ordinary occurrence, the management of which requires going beyond present and local technical or theoretical know-how, to acquire new knowledge and to develop and implement ad hoc strategies.

\subsection{Data on fires}

A dedicated survey allowed the identification of a reference set of data on forest fires, available at Alpine scale. Based on those information, a pan-alpine database of fires has been generated, also in cooperation with another Alpine Space Programme project: ALPFFIRS (www.alpffirs.eu). In the database, each fire is described through a broad set of attributes, listed in Table 1. The database contains information on about 82.000 forest fires, with a differentiated level of thematic contents based on regional availability of data. More than 26.000 occurrences are referred to the decade 2000 - 2009, the time span for which each Alpine region was able to provide information on fires. With specific regard to the identification of extreme events, it has to be highlighted that Total Burnt Area is the only quantitative attribute recorded, without significant lacks of numeracy, in all the fire dataset available at Alpine level. As a consequence, it was used as reference attribute for extreme events selection at Alpine scale. The $99^{\text {th }}$ percentile of the values of the parameter was used for extreme events identification and 105 ha resulted as Alpine threshold for extreme occurrences selection. This kind of approach has a relevant drawback: extreme fires should be better identified in connection with an integrated evaluation of a broad set of variables (dealing, e.g., with territorial and environmental conditions, fire propagation and overall impacts). On the other hand, it allows to make comparisons between different Alpine regions, monitor the spatial and temporal evolution of extreme occurrences and make quantitative evaluations regarding ongoing trends. The analysis at Alpine scale were integrated with a set of regional deepening. It is well known, in fact, that forest fires have distinctive features depending on local conditions. A fire perceived as an exceptional occurrence in one region could fall into a normal regime of events in a different place. Therefore, the different co-operating Alpine regions were asked to make local selections of extreme fire occurrences, based on the whole set of data (both temporal and thematic) available and by adopting locally suitable methodologies. 


\begin{tabular}{|c|c|c|}
\hline Attribute & Notes & Domain \\
\hline \multicolumn{3}{|l|}{ Year } \\
\hline Date & Of the signaling & \\
\hline \multirow{2}{*}{ Season } & April-October: summer & \\
\hline & November-March: winter & \\
\hline X coordinate & \multirow{2}{*}{ Of the ignition point. UTM WGS84 } & \\
\hline Y coordinate & & \\
\hline \multirow{3}{*}{ Cause } & & 1. natural \\
\hline & & 2. doubt \\
\hline & & 3. anthropogenic \\
\hline NUTS2 & \multirow{2}{*}{ Of the ignition point } & \\
\hline NUTS3 & & \\
\hline \multirow{4}{*}{ Altitude } & \multirow{4}{*}{ Of the ignition point } & 1. $0-500 m$ \\
\hline & & $2.500-1.000 m$ \\
\hline & & $3.1 .000-1.500 m$ \\
\hline & & 4. "/> 1.500m \\
\hline \multicolumn{3}{|l|}{ Total Burnt Area } \\
\hline \multicolumn{3}{|l|}{ Forest Burnt Area } \\
\hline \multicolumn{3}{|l|}{ Non Forest Burnt Area } \\
\hline \multirow{2}{*}{ Aspect } & \multirow{2}{*}{$\begin{array}{l}\text { Of the ignition point. Derived from } \\
\text { DTM }\end{array}$} & \\
\hline & & \\
\hline Vegetation Unit & $\begin{array}{c}\text { Of the ignition point. Derived from } \\
\text { the "Map of Natural Vegetation of } \\
\text { Europe" }\end{array}$ & $\begin{array}{l}\text { The legend has been derived from } \\
\text { the "Map of Natural Vegetation of } \\
\text { Europe" report and structured in } \\
\text { three different levels }\end{array}$ \\
\hline \multicolumn{3}{|l|}{ Data Owner } \\
\hline \multicolumn{3}{|l|}{ Mail of the Data Owner } \\
\hline \multicolumn{3}{|l|}{ Data Provider } \\
\hline Mail of the Data Provider & & \\
\hline
\end{tabular}

Table 1. Attributes of the pan-alpine database of fires

\subsection{Data on biotic and other abiotic factors}

Whilst data on fires are basically comparable between the different areas of the Alps (at least for the core set of attributes gathered in the pan-alpine database), information on pathogens, windthrows and heavy snows are characterized by high heterogeneity in data availability between the different Alpine regions. In fact:

- some areas do not run dedicated monitoring systems at all;

- in other regions data are available only in paper format, which makes their acquisition and use difficult in the framework of a project with limited temporal duration;

- even in areas with information available, the categories of data acquired are highly heterogeneous both in terms of thematic contents (e.g. some regions monitor loggings and other regions monitor damages) and spatial/temporal resolution of the monitoring systems. 
Given the high unhomogeneities in the data available at the different Alpine regions, data on biotic and abiotic factors have been introduced in distinct regional sub-sections. They are not conceived to build a comprehensive Alpine picture of the investigated phenomena and do not allow comparisons between the different regions. Moreover, whilst for fires the provided data allowed the definition of an overall Alpine threshold which was estimated with the aim to identify extreme events, information available on biotic and abiotic factors showed not to be suitable for a quantitative selection of extreme events. Besides the heterogeneity of the data, it must be highlighted that information on biotic and abiotic factors are often acquired on yearly basis (e.g. overall loggings or damages due to a certain disturbance), thus making difficult or impossible to link the values to one or more specific occurrences. With the aim to identify the most relevant occurrences a questionnaire has been produced, that partner spread, in their reference regions, to make a list of memory sifted events that, in the last decades, local population, authorities or practitioners perceived as "non-ordinary" or extreme. The questionnaire (Figure 2) asks for a basic set of information: area interested by the event, year or time-span in which the event occurred, stress category (biotic or abiotic), damage factor, main forest cover interested, overall damage (expressed as overall area or damaged volume), eventual further documentation and comments.

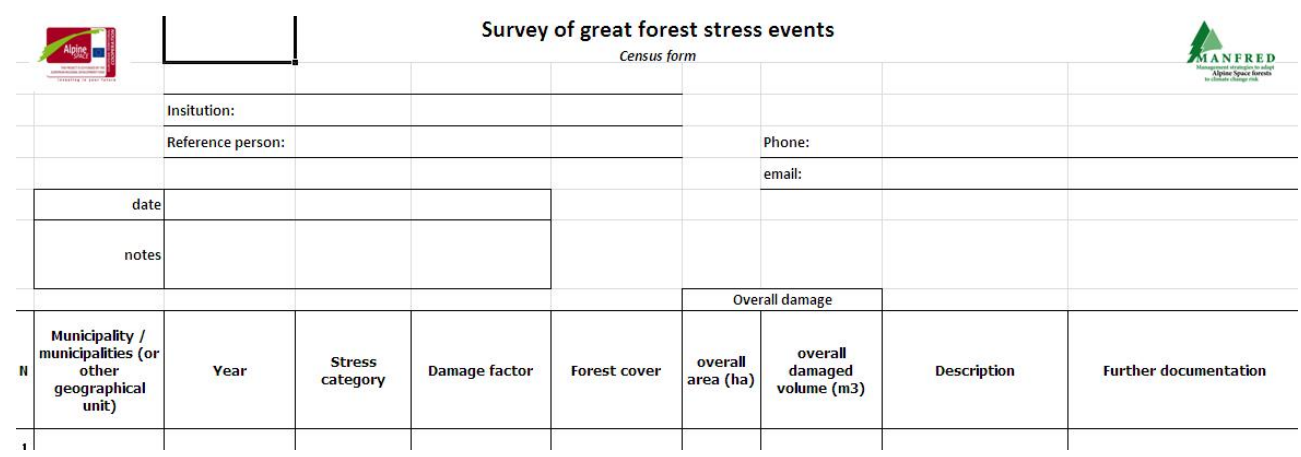

Figure 2. Structure of the questionnaire used to make a census of "non-ordinary" biotic and abiotic events occurred in the last decades in the Alps

In spite of the relevant drawback of such an approach, consisting in the high subjectivity in the process of signaling the events, it must be pointed out that it allowed to carry out the first geo-referenced census available at Alpine level related to 'non-ordinary' or extreme biotic and abiotic occurrences that affected forests in the Alps.

\section{Structure and contents of the platform}

From a technological point of view, the platform has been developed using J2EE (Java enterprise edition) technology. It runs on an Application Server Tomcat 6.0 and uses a db server PostgreSQL 8.4 and PostGIS 1.5 
The webGIS platform consists of three distinct sections (Figure 3) dedicated to:

- fires;

- biotic factors;

- abiotic factors.
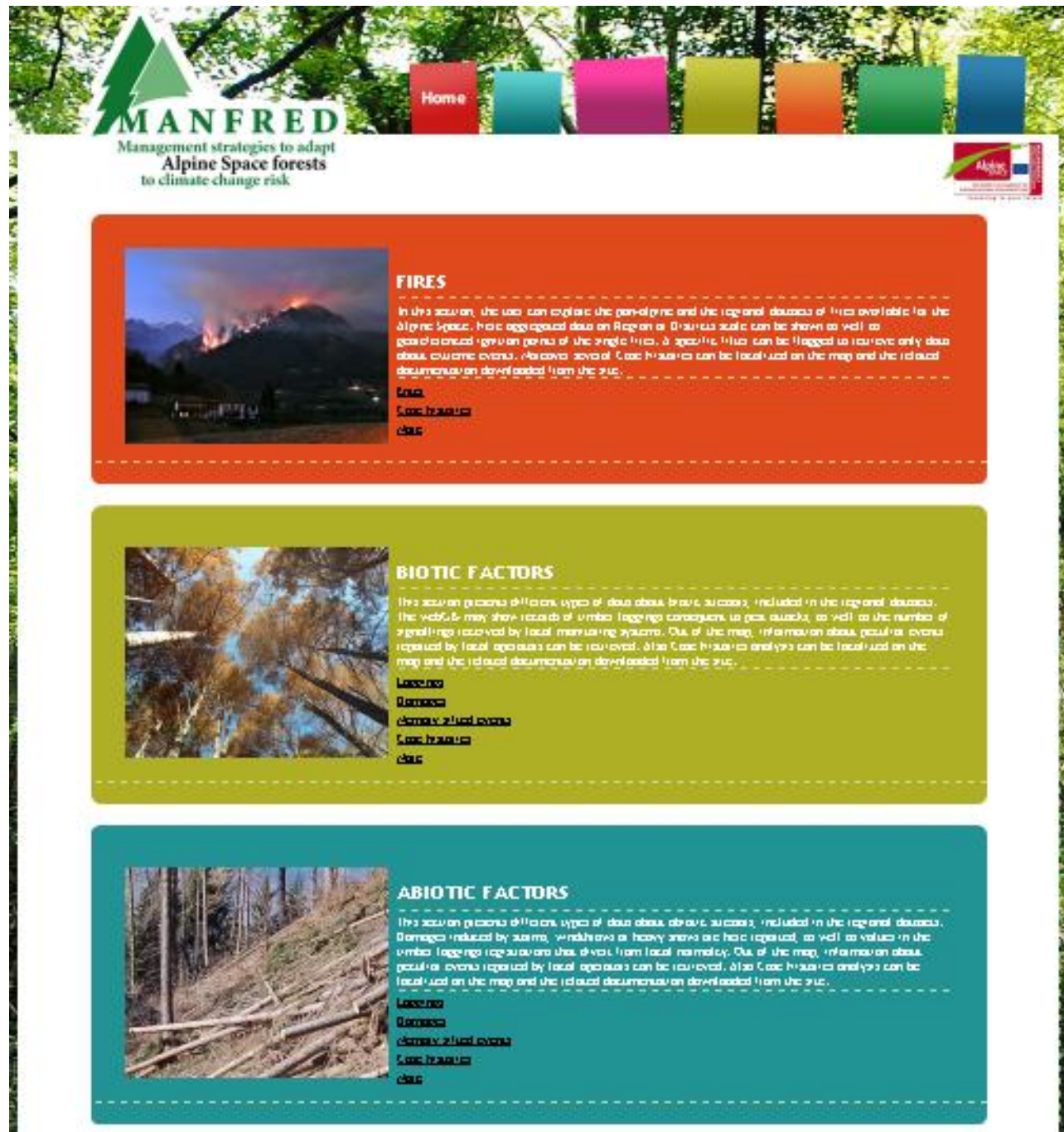

Figure 3. The main sections of the webGIS platform 
Each section contains:

- a set of geographical data;

- Alpine and/or regional reports conceived to summarize and comment the data published;

- reports containing the results of the analysis on the case histories.

It must be pointed out that all the reports produced are based on reference schemes developed for the specific purposes of the project.

\subsection{Fires section}

It allows to work on three reference dataset:

- Alpine;

- Regional;

- Case histories.

When the Alpine option is activated from the Reference dataset box, the platform allows to query and map the data of the pan-alpine database of fires (decade 2000 - 2009) at three aggregation levels: NUTS2 (Region), NUTS3 (District) or it is possible to visualize the distribution of the ignition points of the single fires (Figure 4). Data can be queried based on many of the attributes of the database (Date, Cause, Class of altitude, Aspect, Class of extension) and it is possible to map the results of the queries both based on the overall number of fires or on the overall Burnt Area. In the resulting maps, grey areas evidence the territories for which a specific kind of information is not available. Besides working on the overall set of occurrences, the platform is also conceived to perform queries and produce maps specifically referred to extreme occurrences. The Identify button can be used to obtain, from the maps, analytical information on the results of the queries and the detail of both the Data Owner and the Data Provider. The Legend tool optimizes the visualization of data on the map, based on each query performed. When single fire events are visualized, the legend classes are pre-defined and intended to distinguish ordinary from "extreme" (>105 ha) Alpine occurrences. The Download area hosts a dedicated report summarizing the main results of the analysis carried out on the pan-alpine database. Specifically: state of the art on data availability, descriptive statistics of the overall dataset and of extreme occurrences selection, results of a fire selectivity analysis carried out with the aim to evidence ongoing trends in fire patterns at Alpine scale and results of a deepening performed to identify the Fire Danger Indexes better performing in predicting extreme fire occurrences in the Alps.

When the Regional option is selected in the Reference dataset box, the platform forces to choose (Zone box) a Region. Once the Region has been selected, the system allows to visualize and query (based on the same attribute categories of the previous section) the occurrences that the different regional partners have considered as extreme, using the whole set of data (both from a temporal and thematic perspective) locally available on fires (Figure 5). The Download area hosts the reports that regional partners have produced to comment the results of the analysis on local extreme occurrences and the set of data introduced in the webGIS platform. 


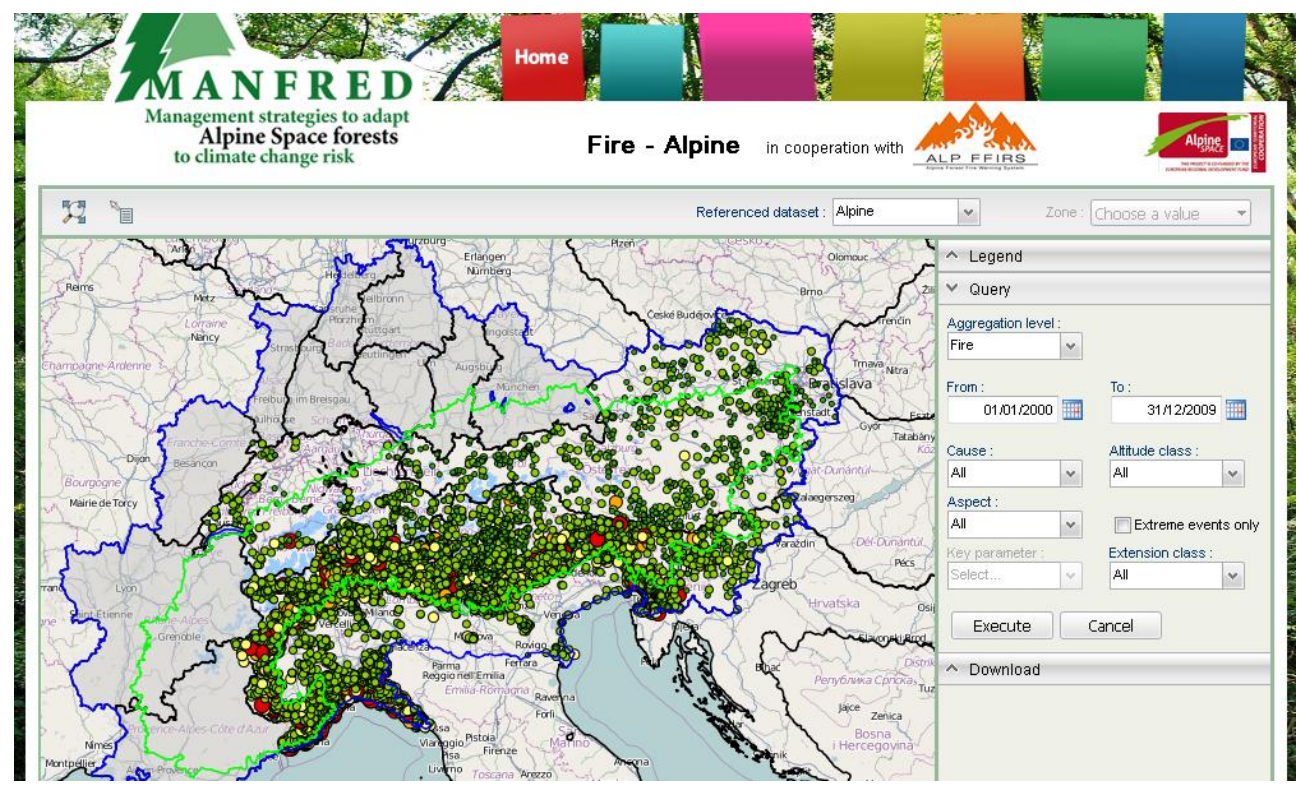

Figure 4. Fire section of the webGIS platform: querying and mapping environment of the Alpine sub-section

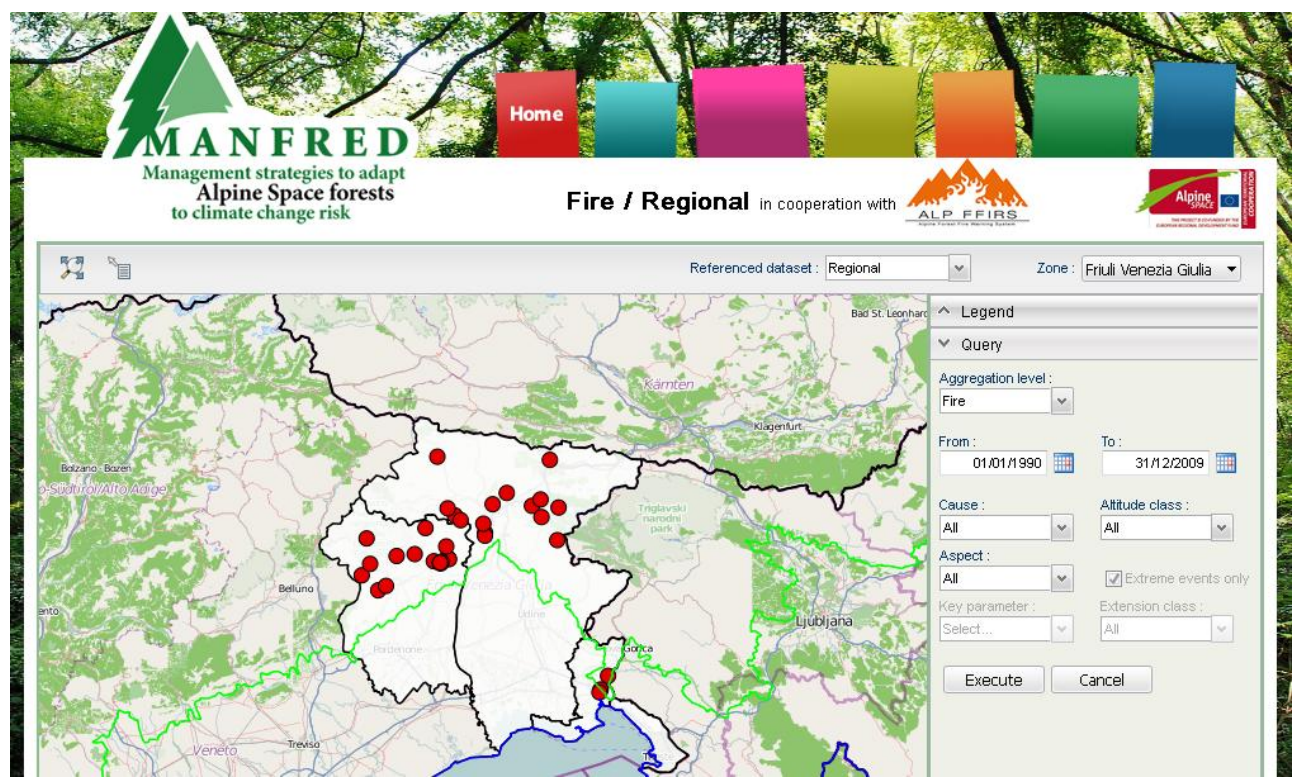

Figure 5. Fire section of the webGIS platform: querying and mapping environment of the Regional sub-sections 
Last area of the fire section is the one dedicated to Case histories. When this option is activated, the platform opens an Alpine map, with the spatial distribution of the fire case histories investigated in the project. The Identify button can be used to obtain basic information on each case history and to download the reports on them carried out by the partners (Figure 6).

The following figures (Figure 7 and Figure 8) provide general schematizations of the Alpine and regional fire sections. For each of them, they give an indication of the main querying options and functionalities.

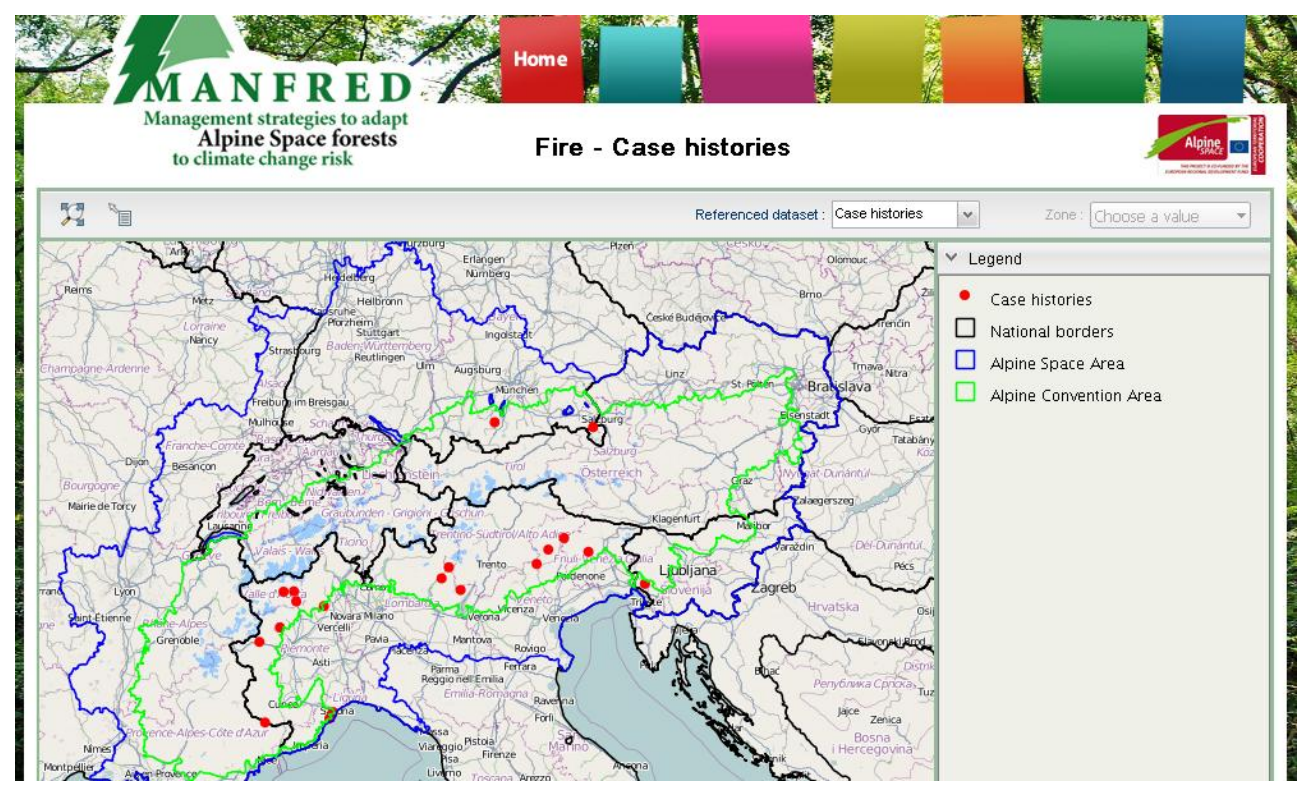

Figure 6. Fire section of the webGIS platform: querying and mapping environment of the Case histories sub-section 


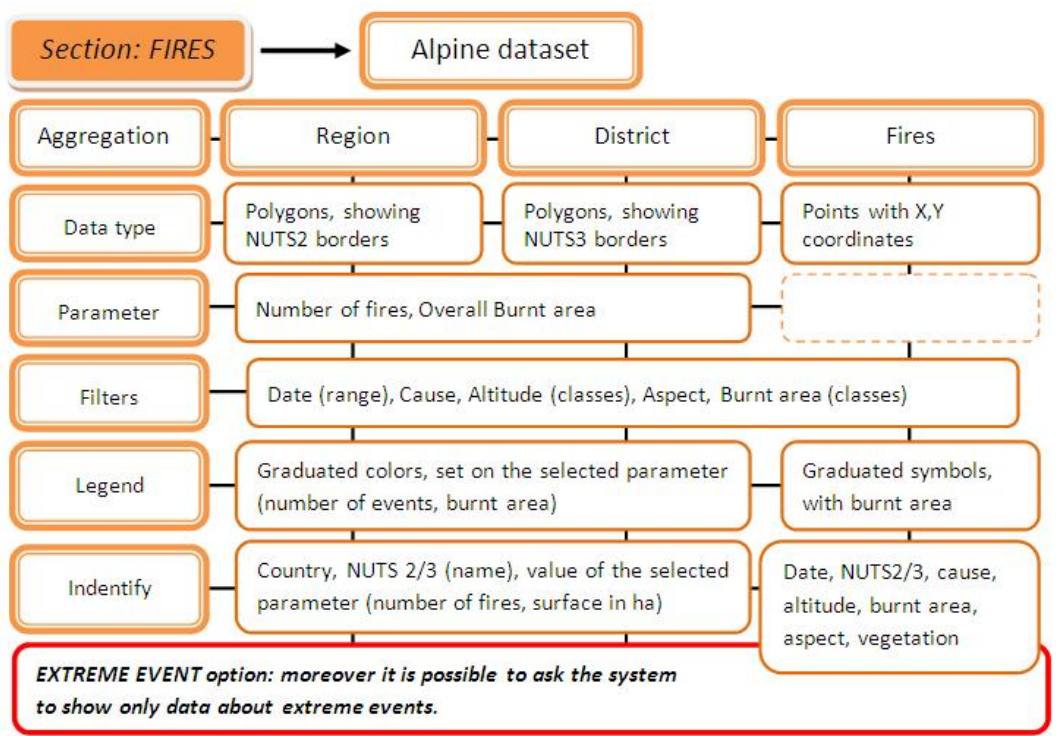

Figure 7. General schematization of the Alpine fire section, with its main querying options and functionalities

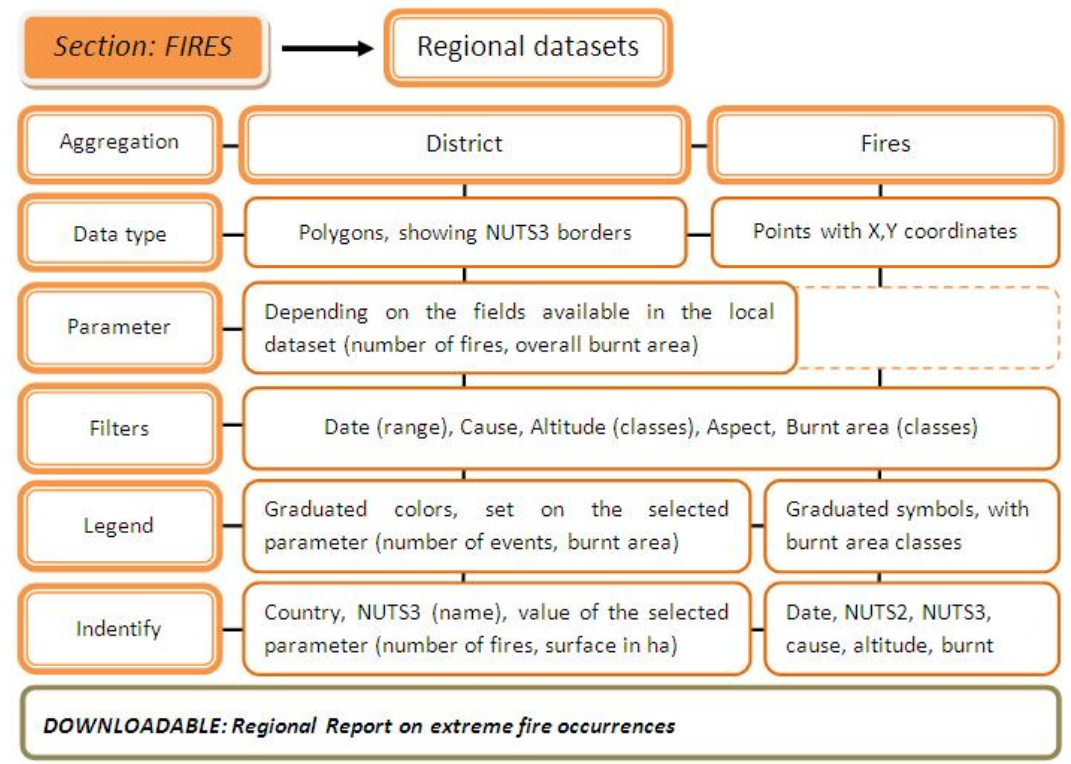

Figure 8. General schematization of the regional fire sections, with their main querying options and functionalities 


\subsection{Biotic and abiotic factors section}

These sections have the same structure and can be considered comparable from an architectural point of view. They respectively contain data and information on pathogens, windthrows and heavy snows.

They work on four reference set of data:

- Loggings;

- Damages;

- Memory Sifted Events;

- Case Histories.

The Loggings sub-section is conceived to visualize, for the regions with available data on loggings at the level of forest parcel, the distribution of the parcels that, on a yearly basis, have been characterized by "non-ordinary" logging levels (Figure 9). For the selection process a dedicated procedure has been applied which was developed by partners in the framework of the project. The procedure integrates yearly data on loggings at parcel level and information on the area of the parcels. It has been intended to minimize the influence of parcels extension in the identification of outlying logging occurrences and to make the different regional distributions of logging data in fact comparable, by means of a normalization process. Besides allowing to show the distribution of outlying parcels, the platform gives the possibility to perform queries at District level, based on the date of loggings and, when available, on the specification of the damaging agent.

The Download Area hosts the reports produced by regional partners to summarize and comment the data introduced in the platform.

The following figure (Figure 10) provides a general schematization of the sub-sections dedicated to loggings both in the biotic and abiotic factors sections.

The Damages sub-section allows to map and query data on any Indicator (e.g. loggings, damaged area, number of signaling), provided by the partners regions, useful to describe the spatial and temporal (yearly basis) evolution of the "pressure" of biotic and abiotic agents on local forests. The platform is conceived to query and map the data, at NUTS2 (Region) or NUTS3 (District) level, based on a broad set of parameters: time-span, various levels of thematic information on the damaging agent (e.g., for pathogens, Category, Order, Family, Species) and querying Key parameter (Figure 11).

Like in the previous case, a dedicated area can be accessed to download regional reports commenting and summarizing the data hosted by the platform.

The Case Histories sub-section is intended to allow the user to visualize, on an Alpine map, the distribution of case histories investigated in the framework of the project. Through the Identify button, the user can obtain basic information on each case history and download the dedicated report produced on the specific occurrence. 


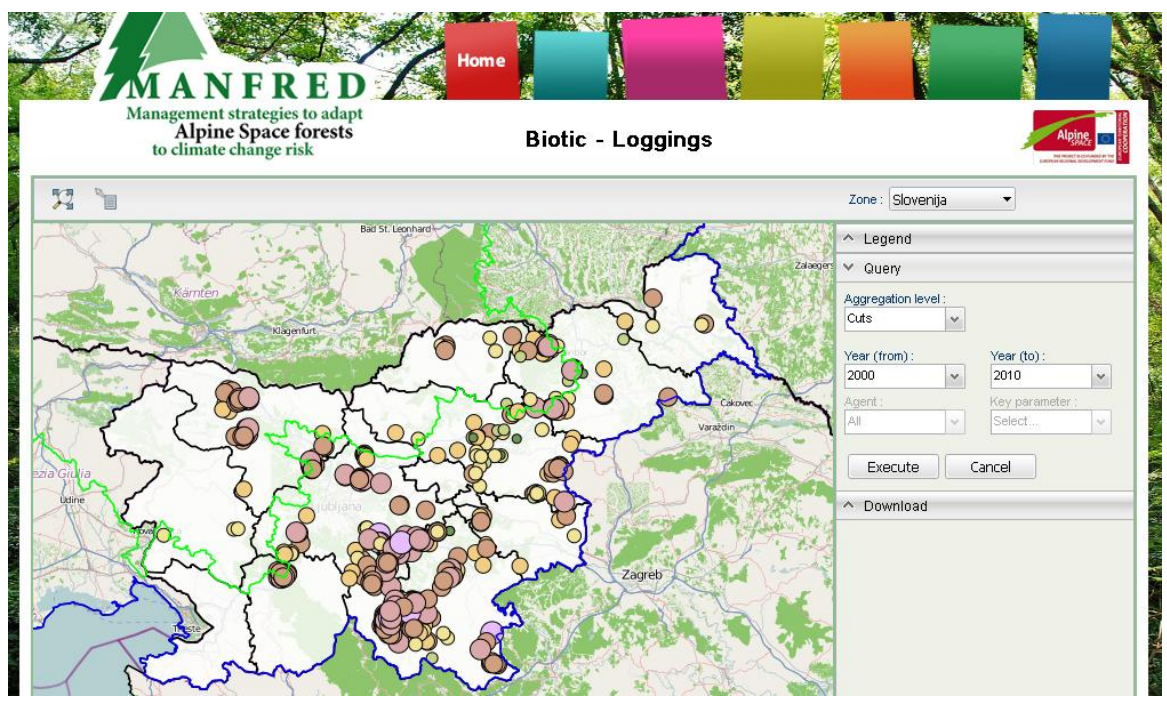

Figure 9. Sections dedicated to Biotic factors and Abiotic factors of the webGIS platform: querying and mapping environment of the Loggings sub-section

Sections: BIOTIC ABIOTIC
Select the country and possibly the region to access the specific subset
NO ALPINE DB AVAILABLE!
Filters
Legend
Identify

DOWNLOADABLE: Regional report on pests and biotic extreme occurrences, Regional

Report on windthrows, snow damages and abiotic extreme occurrences

Figure 10. General schematization of the sub-sections on logging both in the section dedicated to biotic and abiotic factors. Main querying options and functionalities are evidenced 


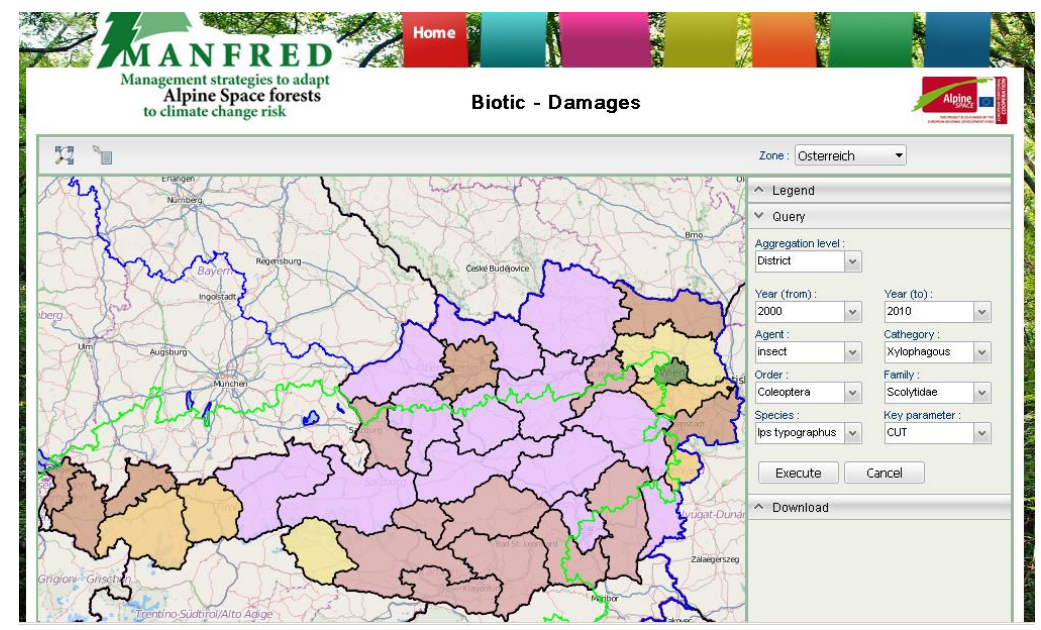

Figure 11. Sections dedicated to Biotic factors and Abiotic factors of the webGIS platform: querying and mapping environment of the Damages sub-section

The following figure (Figure 12) provides a general schematization of damages area, both in the biotic and abiotic factors sections.

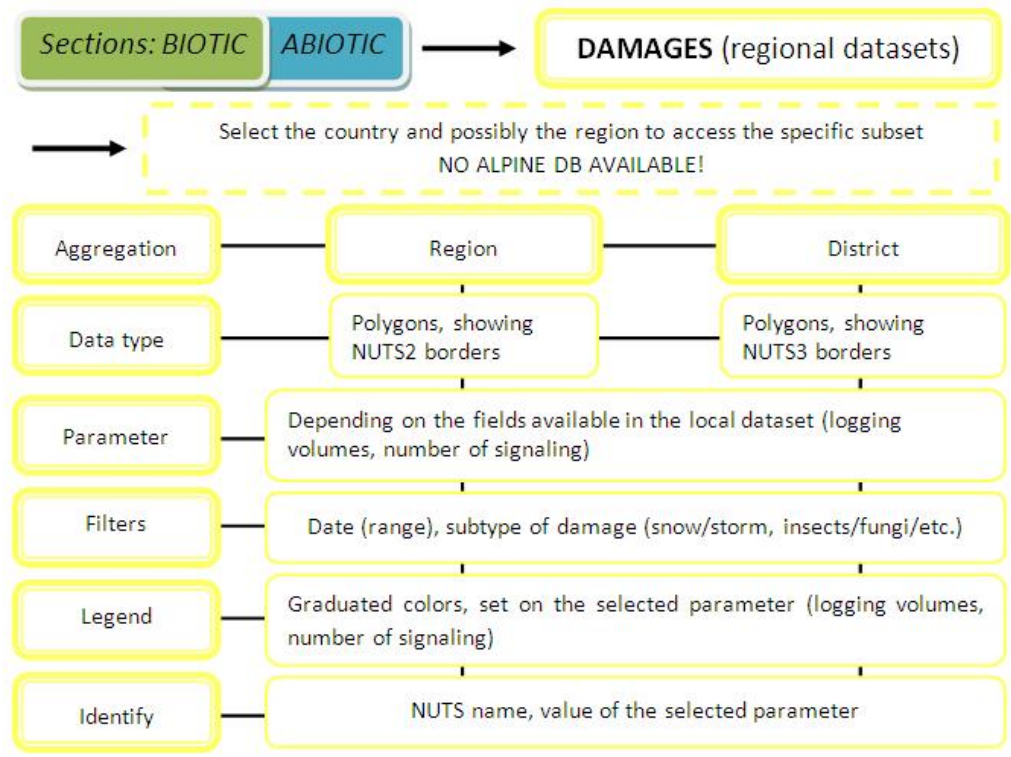

Figure 12. General schematization of the sub-sections on damages both in the section dedicated to biotic and abiotic factors. Main querying options and functionalities are evidenced 
The Memory Sifted Events sub-section has been conceived to allow the user to query the database generated through the spread of the questionnaire used to make a census of the events perceived as "non-ordinary" or extreme. The sub-section gives access to a page conceived to allow the query (not the mapping) of the database, based on District, Damage factor and Year of occurrence of the events. The system lists the occurrences meeting the querying rules, with the specification of the whole set of details introduced in the questionnaire (Figure 13).
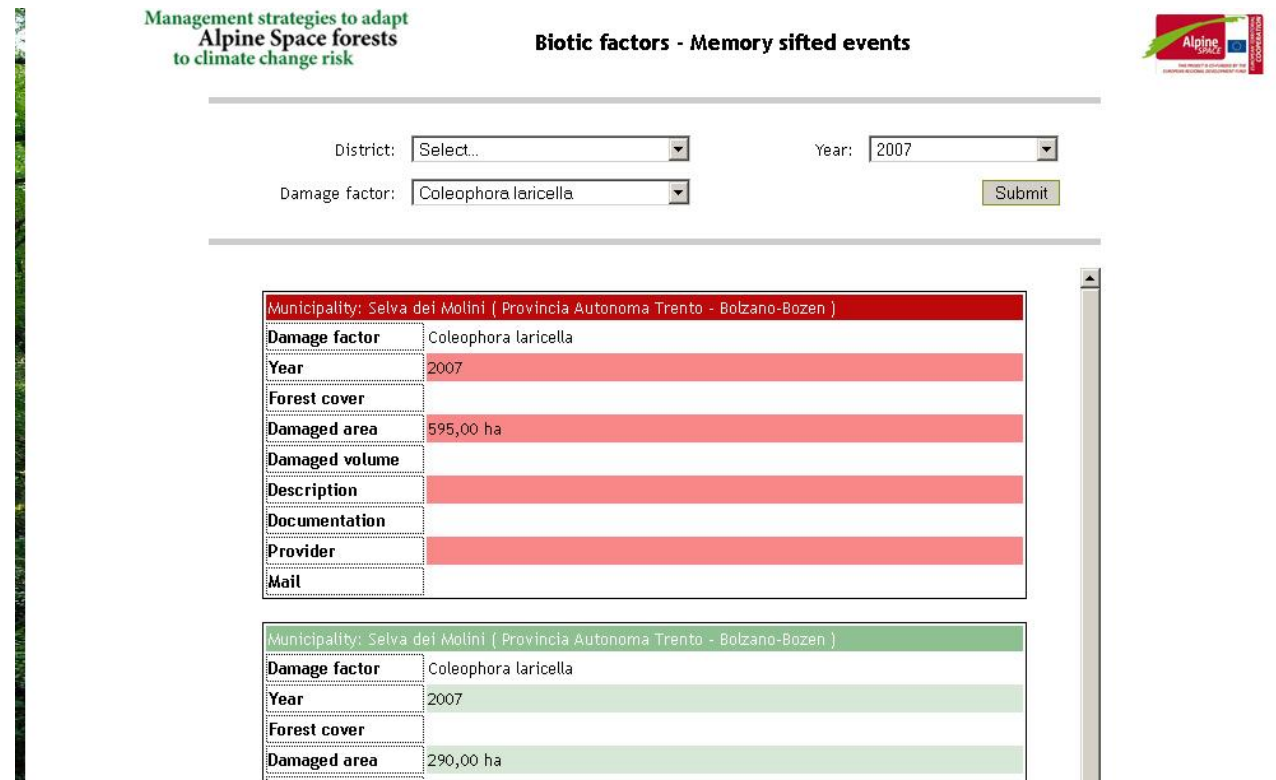

Figure 13. Sections dedicated to Biotic factors and Abiotic factors of the webGIS platform: querying environment of the Memory Sifted Events sub-section

\section{Conclusions}

Building a webGIS within the MANFRED project had different goals. Obviously, to contain and to make easily accessible the vast dataset gathered during the three years of the project among several partners, giving body to the effort of collecting a common knowledge base, provided by the several research centers and institutions of all the involved countries. The webGIS provides, in the form of an interactive reference book, the useful key to the consultation and interpretation of those data. It also gives an overall picture of the situation regarding forest damages monitoring procedures on the Alpine scale. Both the database and the webGIS are intended to be open-ended activities, to be further improvable, and to be run steadily as a web resource. The possibility to locate and retrieve information about extreme events occurred in the recent past can be particularly meaningful for local administrators and involved practi- 
tioners, who could find themselves in the necessity to deal with new similar events due to fire, snow, storm or pests within an Alpine forest environment. To this purpose, contact details of the main referents for the case histories have been made available in the webGIS. We remark here the importance to confront each others on our experiences from the recent past and on the strategies we developed to manage them, both assimilating the good practices and learning from errors. We believe, in fact, that the most effective way to face the uncertainties related to climate change is to have at disposal the widest and most various range of knowledge, enriched by the contribute of everyone between the two ends of the Alps and beyond. This research made the contacts between several experts and professionals from the six countries that have been involved. These actions have hopefully created a stable network of people and of shared knowledge: a wealth in resources that we wish to be durable and to be able to bear fruit in the preservation and the future management of our forests and our communities.

With specific regard to the data gathered and the investigations carried out, it is important to remark that:

- the pan-alpine database of fires is probably, today, the most complete set of information on forest fires available at Alpine level;

- the census of memory sifted events represents the first geo-referenced census available at Alpine level related to 'non-ordinary' biotic and abiotic occurrences that affected forests in the Alps;

- all the case histories (\#34) and the main contents of Alpine and regional reports (\#20) have been integrated in a document (Handbook) titled "Extreme stress events in the Alpine forests: management experiences based on recent occurrences", available in the MANFRED project website.

Based on the work done and taking the difficulties experienced in collecting and integrating the different set of data available at Alpine level as a starting point for mentioning some relevant future challenges, we can underline that it would be strategic, with the aim to build an integrated picture on the state of health of forests in the Alps, to improve the existing monitoring tools and steer the acquisition of data towards a higher degree of homogeneity between the different Alpine regions. Moreover, the opportunity to run permanent monitoring systems on the Alpine forests should be recognized and, hopefully, the platform generated in the framework of MANFRED project should be further integrated and updated in the future.

\section{Acknowledgements}

This work was conducted under the European Project MANFRED, funded by the European Regional Development fund of the Alpine Space Program, reference number 15-2-3-D (MANFRED).

A large set of partners co-operated in the activities: ERSAF. Ente Regionale per i Servizi all'Agricoltura e alle Foreste; FVA. Forstliche Versuchs - und Forschungsanstalt BW; Slovenian 
Forest Service; Slovenian Forest Institute; Catholic University of Brescia; Italian Ministry of the Environment, Land and Sea; University of Camerino; CEMAGREF. Institut de recherche pour l'ingénierie de l'agriculture et de l'environnement; IPLA. Istituto per le Piante da Legno e l'Ambiente; Regione Autonoma Valle d'Aosta; LWF. Bavarian State Institute of Forestry; WSL. Swiss Federal Institute for Forest, Snow and Landscape Research; BOKU. University of Natural Resources and Life Sciences; BFW. Research and Training Centre for Forests, Natural Hazards and Landscape.

External contributions were provided by the University of Torino and by Regione Autonoma Friuli Venezia Giulia.

The research and its publication have been funded (partially or fully) by the Catholic University of the Sacred Heart, in the framework of its programs aimed at the promotion and the dissemination of scientific research.

\section{Author details}

Stefano Oliveri ${ }^{{ }^{*}}$, Marco Pregnolato ${ }^{2}$ and Giacomo Gerosa ${ }^{2}$

*Address all correspondence to: stefano.oliveri@ecometrics.it

1 Ecometrics srl., Italy

2 Catholic University of Brescia, Mathematics and Physics Department, Italy

\section{References}

[1] Faccoli, M, \& Battisti, A. in press) Climate change and forest pests: models from the Alps- Canadian Journal of Forest Research

[2] Marini, L, Ayres, M. P, Battisti, A, \& Faccoli, M. Climate affects severity and altitudinal distribution of outbreaks in an eruptive bark beetle. Climatic Change (2012). DOIs10584-012-0463-z.

[3] Lindner, M, Maroschek, M, Netherer, S, et al. Climate change impacts, adaptive capacity, and vulnerability of European forest ecosystems. For Ecol Manag (2010). , 259, 698-709.

[4] Boisvenue, C, \& Running, S. W. Impacts of climate change on natural forest productivity-evidence since the middle of the 20th century. Glob Change Biol. (2006). , 12, 1-21. 
[5] Battisti, A, Stastny, M, Netherer, S, Robinet, C, Schopf, A, Roques, A, \& Larsson, S. Expansion of Geographic Range in the Pine Processionary Moth Caused by Increased WinterTemperatures. Ecological Applications (2005). , 15(6), 2084-2096.

[6] Battisti, A. Forests and climate change- lessons from insects. Forest@ (2004). http:// www.sisef.it/accessed 15 June 2012), 1(1), 17-24.

[7] Faccoli, M. Effect of Weather on Ips typographus (Coleoptera Curculionidae) Phenology, Voltinism, and Associated Spruce Mortality in the Southeastern Alps. Environ. Entomol. (2009). , 38(2), 307-316.

[8] Battisti, A, Stastny, M, Buffo, E, \& Larsson, S. A rapid altitudinal range expansion in the pine processionary moth produced by the 2003 climatic anomaly. Global Change Biology (2006). DOIj.1365 2486.2006.01124.x, 12, 662-671.

[9] MANFRED project Official websitewebGIS platform area. www.manfredproject.eu/ webgisaccessed 13 July (2012). 
